\title{
High energy spatially radiative properties of Vela X pulsar wind nebula
}

\author{
Fang-Wu Lu ${ }^{1,2}$, Quan-Gui Gao ${ }^{1,2}$, Bo-Tao Zhu ${ }^{2}$, and Li Zhang ${ }^{2}$ \\ 1 Department of Physics, Yuxi Normal University, Yuxi 653100, PR China \\ 2 Department of Astronomy, Key Laboratory of Astroparticle Physics of Yunnan Province, Yunnan University, \\ Kunming 650091, PR China \\ e-mail: lizhang@ynu.edu.cn
}

Received 26 September 2018 / Accepted 13 March 2019

\begin{abstract}
Context. Vela $\mathrm{X}$ is a middle-aged pulsar wind nebula $(\mathrm{PWN})$ that has been detected in radio, $\mathrm{X}$-ray, and $\gamma$-ray bands. The observations indicate that that photon index and flux density of the inner regions of the nebula have obvious changes as the radial distance increases in the X-ray band, and the surface brightness has been discovered to decrease with the increase of radial distance in $\gamma$-ray band.

Aims. The multi-band photon emission and high energy spatially radiative properties of Vela X are investigated in the framework of a spatially dependent particle transport model.

Methods. Electron distribution inside the PWN was described by a spatially dependent particle transport equation and the evolution of the non-thermal photon was described as a photon conservation equation under the assumption of a spherically symmetric system with dynamical evolution. In this model, the dynamical and particle evolution equations were simultaneously solved, and the $\gamma$-ray attenuation by the supernova remnant (SNR) photon fields in the PWN were taken into account.

Results. The observed spectral energy distributions of Vela X, including X-ray spectra of the inner regions and $\gamma$-ray spectra observed at $\mathrm{GeV}$ and $\mathrm{TeV}$ bands, are well reproduced. The radial variations of the photon index in $\mathrm{X}$-ray band and the surface brightness in the $\mathrm{TeV}$ energy range as well as the $\gamma$-ray spectra of the inner region and ring extension can also be well reproduced in the framework of our model. The spatial variations of photon indices at $\mathrm{GeV}$ and $\mathrm{TeV}$ bands are predicted and show that the photon index in $\mathrm{GeV}$ band increases with the increase of radial distance while there is only a slight change in the $\mathrm{TeV}$ band. Moreover, the modelling results reveal that the $\gamma$-ray attenuation by the SNR photon fields is important for modifying very high energy $\gamma$-ray spectrum of Vela X.
\end{abstract}

Key words. pulsars: general - ISM: individual objects: Vela X - radiation mechanisms: non-thermal

\section{Introduction}

Vela $\mathrm{X}$ is a middle-aged pulsar wind nebula (PWN) that is powered by the Vela pulsar (PSR B0833-45; Weiler \& Panagia 1980), and located in the centre of the Vela supernova remnant (SNR). As one of the closest pulsars to the Earth, the Vela pulsar has a period of $P=89.3 \mathrm{~ms}$ (Large et al. 1968) and a period derivative of $\dot{P}=1.25 \times 10^{-13} \mathrm{~s} \mathrm{~s}^{-1}$ (Lyne et al. 1996), which implies that the current spin-down luminosity of this pulsar has a value of $L_{0}=6.9 \times 10^{36} \mathrm{erg} \mathrm{s}^{-1}$ and a characteristic age of $\tau_{\mathrm{c}}=11.3 \mathrm{kyr}$ (Taylor et al. 1993; Lyne et al. 1996). The value of distance to the Vela SNR is estimated to be $290 \mathrm{pc}$ according to the radio and optical parallax measurements (Caraveo et al. 2001; Dodson et al. 2003), and the age is usually considered to be the characteristic age of the Vela pulsar (Reichley et al. 1970; Manzali et al. 2007). Lyne et al. (1996) estimated that the braking index for the Vela pulsar has a value of $n=1.4$. However, based on the new long-term braking index measurements, Espinoza et al. (2017) suggested that the value of the braking index of the Vela pulsar is $n=1.7$, which is adopted in this paper.

The photon emission from Vela $\mathrm{X}$ has been detected at radio, $\mathrm{X}$-ray, and $\gamma$-ray bands, and showed that the nebula is a morphologically complex source. At radio and $\mathrm{GeV}$ energy ranges, Vela $\mathrm{X}$ shows a filamentary structure usually referred to as the halo (e.g. Abdo et al. 2010; Hinton et al. 2011; Grondin et al.
2013). In the radio band, Alvarez et al. (2001) revealed that the PWN has a power-law spectral index of $0.39 \pm 0.03$ in the $30-8400 \mathrm{MHz}$ energy range. In the GeV $\gamma$-rays, according to the Fermi Large Area Telescope (LAT) observations, Abdo et al. (2010) revealed that the non-thermal spectrum of Vela X can be well fitted by a power law with a photon index of $2.41 \pm 0.09 \pm$ 0.15 in the $0.2-20 \mathrm{GeV}$ energy range, and Grondin et al. (2013) pointed out that the photon spectrum above $0.2 \mathrm{GeV}$ can be well described by a smoothly broken power law with spectral indices of $\Gamma_{1}=1.83 \pm 0.07 \pm 0.27, \Gamma_{2}=2.88 \pm 0.21 \pm 0.06$ and a break energy of $E_{\mathrm{b}}=2.1 \pm 0.5 \pm 0.5 \mathrm{GeV}$. In the X-ray band, the ROAST observations revealed a much smaller emission region usually called the cocoon. (Markwardt \& Ögelman 1995), and the Chandra X-ray images showed a double torus and jet-like structure at the centre of the PWN (Helfand et al. 2001). The ROAST and ASCA data found that the power-law photon index in the X-ray band has a value of 2.1 (Markwardt \& Ögelman 1995, 1997), and the XMM-Newton data showed that the photon index is 2.3 (LaMassa et al. 2008). In the inner regions of the nebula, based on the XMM-Newton/MOS and BeppoSAX/MECS data, Mangano et al. (2005) pointed out that the power-law photon index and flux density in the $3-10 \mathrm{keV}$ energy range have obvious changes as the radial distance increases. In the $\mathrm{TeV}$ $\gamma$-rays, the photon emission region, which has been detected by High Energy Stereoscopic System (H.E.S.S.), is coincident with the radio halo and X-ray cocoon. (Aharonian et al. 2006; 
Abramowski et al. 2012). Aharonian et al. (2006) have shown that the energy spectrum of Vela $X$ in the $0.55-65 \mathrm{TeV}$ energy range can be well fitted by an exponentially cut-off powerlaw function, with a photon index of $1.45 \pm 0.09_{\text {stat }} \pm 0.2_{\text {sys }}$ and an exponential cut-off at an energy of $13.8 \pm 2.3_{\text {stat }} \pm$ $4.1_{\text {sys }} \mathrm{TeV}$. While according to the deeper observation of the entire Vela X nebula region with H.E.S.S., Abramowski et al. (2012) revealed that the total differential energy spectrum in the energy of $0.75-70 \mathrm{TeV}$ can be well described with a hard photon index of $1.32 \pm 0.06_{\text {stat }} \pm 0.12_{\text {sys }}$ and a cut-off energy of $14.0 \pm 1.6_{\text {stat }} \pm 2.6_{\text {sys }}$ TeV. Moreover, the H.E.S.S. Collaboration reported that the energy averaged surface brightness of Vela $\mathrm{X}$ decreases with the increase of radial distance in the $\mathrm{TeV} \gamma$-ray band (Abramowski et al. 2012).

To interpret the non-thermal radiative properties of Vela X, various models have been proposed (e.g. Horns et al. 2006; de Jager et al. 2008; Zhang \& Yang 2009; Abdo et al. 2010; Hinton et al. 2011; Mattana et al. 2011). Under the assumption that the particles in the nebula are composed of electrons and hadrons, Horns et al. (2006) proposed a leptonic-hadronic model, in which the X-ray emission is produced by the synchrotron radiation of relativistic electrons and the $\mathrm{TeV}$ photons are mainly from the decay of neutral pions produced in protonproton collisions inside the nebula. In parallel, de Jager et al. (2008) suggested that there are two distinct electron populations existing in Vela $\mathrm{X}$, one is responsible for the observed spectrum in radio and $\mathrm{GeV} \gamma$-ray bands, and the other for the X-ray and $\mathrm{TeV} \gamma$-ray emission. Both the leptonic-hadronic and leptonic models can well reproduce the non-thermal photon spectrum of Vela X. However, these two models cannot explain the spatial variations of the energy spectrum of the PWN observed at X-ray and $\mathrm{TeV} \gamma$-ray bands, because the distribution of particles in the nebula is assumed to be uniform. In recent years, a spatially dependent particle transport model was proposed to explain the spatial variation properties of PWNe (e.g. Vorster \& Moraal 2013; Porth et al. 2016; Lu et al. 2017a; Ishizaki et al. 2017; van Rensburg et al. 2018), in which the particle number density is spatially dependent and the processes of convection and diffusion are taken into account. Assuming that the PWN is a spherical symmetric system, Porth et al. (2016) studied the radial variations of the photon index and flux density of Vela $\mathrm{X}$ in the X-ray band with a steady-state particle transport model, and found that the spatial variations of photon index and flux density of the inner regions of the nebula can be well reproduced in the framework of their model. However, Porth et al. (2016) did not investigate the multi-band photon emission and spatially radiative properties of $\gamma$-rays of the PWN, because their main aims are to investigate the particle transport and the influence of the diffusion effect on the particle spectrum.

In this paper, we study the multi-band photon emission and high energy spatially radiative properties of Vela $\mathrm{X}$ in the framework of a spatially dependent particle transport model proposed by Lu et al. (2017a). In the model, the object is assumed to be a spherical symmetric system. The X-ray and very high energy (VHE) $\gamma$-ray observations have shown that the Vela X nebula is a highly asymmetric PWN (Markwardt \& Ögelman 1995; Aharonian et al. 2006; Abdo et al. 2010). However, the X-ray observations of the inner region of Vela $X$ show a bright X-ray nebula that is approximately spherically symmetric (Mangano et al. 2005; Mattana et al. 2011). Besides, the multiwavelength maps of the analysis region of Vela $\mathrm{X}$ are usually treated as a circular region (e.g. Aharonian et al. 2006; Abdo et al. 2010; Abramowski et al. 2012; Tibaldo et al. 2018). Thus, as a first-order approximation, the spatially dependent and spherical symmetric model can help us to study the spatial variations of azimuthally integrated spectra in the Vela PWN. The organization of the paper is as follows. In Sect. 2, the spatially dependent particle transport model is simply described and the modelling results are presented. The main conclusions and discussions are given in Sect. 3.

\section{Model and results}

In this section, the spatially dependent particle transport model of Lu et al. (2017a) will be briefly reviewed, and the modelling results of Vela $X$, including the spectral energy distributions (SEDs), radial variations of photon index, and flux density of the X-ray emission in the inner regions of the nebula, as well as the spatial variations of photon indices and surface brightness in the $\mathrm{GeV}$ and $\mathrm{TeV}$ bands, will be presented.

\subsection{Model description}

In a spherically symmetric system, the evolution of electron distribution $n_{\mathrm{e}}=n_{\mathrm{e}}(r, \gamma, t)$ inside the PWN can be described with the particle transport equation

$$
\begin{aligned}
\frac{\partial n_{\mathrm{e}}}{\partial t}= & \kappa \frac{\partial^{2} n_{\mathrm{e}}}{\partial r^{2}}+\left[\frac{1}{r^{2}} \frac{\partial}{\partial r}\left(r^{2} \kappa\right)-V\right] \frac{\partial n_{\mathrm{e}}}{\partial r}-\frac{1}{r^{2}} \frac{\partial}{\partial r}\left[r^{2} V\right] n_{\mathrm{e}} \\
& +\frac{\partial}{\partial \gamma}\left[\dot{\gamma} n_{\mathrm{e}}\right]+Q_{\mathrm{inj}}
\end{aligned}
$$

where the processes of convection, diffusion, adiabatic loss, and radiative losses are taken into account. In the above equation, $r$ represent the radial position, $\gamma$ is the Lorentz factor of electrons, $\kappa=\kappa(r, \gamma, t)$ is the particle diffusion coefficient, $V=V(r)$ is the convection velocity, $\dot{\gamma}$ is the total energy loss rate of electrons, and $Q_{\text {inj }}$ is the electrons injected at the termination shock. The number of particles per energy interval that flow through the termination shock should be equal to the number of particles injected from the central pulsar, which implies the inner boundary condition can be expressed as (e.g. Ng \& Gleeson 1975; Vorster \& Moraal 2013)

$V_{0} n_{\mathrm{e}}-\kappa\left(r_{0}(t), \gamma, t\right) \frac{\partial n_{\mathrm{e}}}{\partial r}=\frac{Q_{\mathrm{inj}}(\gamma, t)}{4 \pi r_{0}^{2}(t)}$,

where $r_{0}(t)$ is the radius of the termination shock at time $t$ and $V_{0}$ represents the particle convection velocity at the shock.

According to de Jager et al. (2008), the particles in Vela X are assumed to be composed of two distinct electron populations, one at low energies responsible for the radio and $\mathrm{GeV} \gamma$ ray emission and the second higher energy population for the $\mathrm{X}$-ray and $\mathrm{TeV}$ emission. Thus, the distribution of injected electrons at the termination shock is given by

$Q_{\text {inj }}(\gamma, t)= \begin{cases}Q_{0, \mathrm{R}}(t)\left(\frac{\gamma}{\gamma_{\max , \mathrm{R}}}\right)^{-\alpha_{1}} & \gamma_{\mathrm{min}, \mathrm{R}}<\gamma<\gamma_{\max , \mathrm{R}}, \\ Q_{0, \mathrm{X}}(t)\left(\frac{\gamma}{\gamma_{\max , \mathrm{X}}}\right)^{-\alpha_{2}} & \gamma_{\min , \mathrm{X}}<\gamma<\gamma_{\max , \mathrm{X}},\end{cases}$

where $Q_{0, i}(t)$ is the normalization constant and can be calculated by

$\int_{0}^{\infty} Q_{0, i}(t)\left(\gamma / \gamma_{\max , i}\right)^{-\alpha_{i}} \gamma m_{\mathrm{e}} c^{2} \mathrm{~d} \gamma=\eta_{i} L(t)$.

Here, $\eta_{i}(i=\mathrm{R}, \mathrm{X})$ is the fraction of spin-down luminosity $L(t)$ of a pulsar converted to relativistic particle energy in each respective component. Assuming that the pulsar is a pure dipole radiator with a braking index of $n$, the time evolution of the spin-down 
luminosity of a pulsar can be given by

$$
L(t)=L_{0}\left(1+\frac{t}{\tau_{0}}\right)^{-\frac{n+1}{n-1}}
$$

where $L_{0}$ is the initial luminosity and $\tau_{0}=2 \tau_{c} /(n-1)-T_{\text {age }}$ is the initial spin-down timescale, and $T_{\text {age }}$ is the age of the pulsar. In Eq. (3), $\gamma_{\max , i}$ is the respective maximum energy of the two distinct electron populations, $\gamma_{\min , i}$ is the minimum energy of electrons, which is assumed to be 1.0 in this paper according to de Jager et al. (2008), and $\alpha_{1}$ and $\alpha_{2}=2.0$ are the corresponding spectral indices.

Following Kennel \& Coroniti (1984a,b), the magnetic field in the PWN is assumed to be toroidal, and then the time and spatial evolutions of magnetic field in the nebula can be described as

$$
B(r, t)=B_{0}(t)\left[\frac{r_{0}(t)}{r v(r)}\right]
$$

where $v(r)$ is the radial profiles of the convection velocity, and $B_{0}(t)$ is the magnetic field at the termination shock that is obtained by $W_{\mathrm{B}}(t)=\int_{r_{0}(t)}^{R_{\mathrm{pwn}}(t)} B^{2}(r, t) r^{2} \mathrm{~d} r / 2$, where $R_{\mathrm{pwn}}(t)$ is the radius of the PWN. The function $W_{\mathrm{B}}(t)$ is calculated with $\mathrm{d} W_{\mathrm{B}}(t) / \mathrm{d} t=\eta_{\mathrm{B}} L(t)-\left[W_{\mathrm{B}}(t) / R_{\mathrm{pwn}}(t)\right]\left[\mathrm{d} R_{\mathrm{pwn}}(t) / \mathrm{d} t\right]$ and $\eta_{\mathrm{B}}$ is the fraction of pulsar spin-down luminosity converted to magnetic field energy (e.g. Pacini \& Salvati 1973). According to Lerche \& Schlickeiser (1981), the evolution of the diffusion coefficient is given by

$\kappa(r, \gamma, t)=\kappa_{0}(t)\left(\frac{\gamma m_{\mathrm{e}} c^{2}}{1 \mathrm{TeV}}\right)\left[\frac{r_{0}(t)}{r v(r)}\right]^{-1}$,

and $\kappa_{0}(t) \propto 1 / B(t)$ is the diffusion at the termination shock.

In the model, the evolution of non-thermal photons arising from the radiation processes of relativistic electrons of the PWN is described as a photon conservation equation

$$
\frac{\partial n_{\gamma}}{\partial t}+\frac{n_{\gamma}}{t_{\mathrm{esc}}}=Q_{\gamma}^{\mathrm{syn}}+Q_{\gamma}^{\mathrm{ics}}+L_{\gamma}^{\mathrm{ssa}}+L_{\gamma}^{\gamma \gamma}
$$

In the above equation, $n_{\gamma}=n_{\gamma}(r, x, t)$ is the number density of photons with the dimensionless photon energy $x=E / m_{\mathrm{e}} c^{2}, t_{\mathrm{esc}}$ represents the photon escape timescale, $Q_{\gamma}^{\mathrm{syn}}$ and $Q_{\gamma}^{\mathrm{ics}}$ are the emissivity of synchrotron radiation and inverse Compton process, respectively, $L_{\gamma}^{\text {ssa }}$ is the loss rate for photons caused by the process of synchrotron self-absorption, and $L_{\gamma}^{\gamma \gamma}$ is the lose rate of photon-photon pair production inside the PWN. A more detailed description and numerical calculation of the spatially dependent model are shown in Lu et al. (2017a,b), and the radial variations of surface brightness and photon index at different bands are calculated according to Holler et al. (2012).

The dynamical evolution of the PWN is simulated according to the model presented by Bucciantini et al. (2011). In their model, the object is assumed to be a spherically symmetric system and the proper motion of the pulsar as well as the radiation losses of particles are negligible, and then the dynamical evolution of a PWN is divided into three phases: free-expansion, reverberation, and Sedov-Taylor phases. In the initial free-expansion phase, the radius of the PWN is given by

$$
R_{\mathrm{pwn}}(t)=\left(\frac{E_{\mathrm{sn}}^{3} L_{0}^{2}}{M_{\mathrm{ej}}^{5}}\right)^{\frac{1}{10}}\left(\frac{t}{1+\frac{t}{\tau_{0}}}\right)^{\frac{6}{5}} \frac{1}{1-s} \sum_{i=0}^{\infty} c_{i} s^{i},
$$

where $E_{\mathrm{sn}}$ is the energy of the supernova explosion, $M_{\mathrm{ej}}$ is the mass of the supernova ejecta, $s=\left(t / \tau_{0}\right) /\left(1+t / \tau_{0}\right)$, and the coefficients $c_{i}$ are given in Bucciantini et al. (2004). In the reverberation phase, the dynamical evolution radius is calculated with

$M_{\mathrm{sw}} \ddot{R}_{\mathrm{pwn}}(t)=4 \pi R_{\mathrm{pwn}}^{2}(t)\left[P_{\text {in }}(t)-P_{\text {out }}(t)\right]$.

Here, $M_{\mathrm{sw}}$ is the mass of material swept up by the PWN when it expands inside the SNR, $P_{\text {in }}$ is the pressure of the PWN shell, and $P_{\text {out }}$ is the pressure of the SNR ejecta at the position of the PWN shell. In the Sedov-Taylor phase, the radius is determined by

$R_{\text {pwn }}^{4}\left(t_{\text {Sedov }}\right) P_{\text {in }}\left(t_{\text {Sedov }}\right)=R_{\text {pwn }}^{4}(t) P(t)$,

where $t_{\text {Sedov }}$ is the time at which the Sedov-Taylor phase begins, $P(t)=\rho_{\mathrm{ism}} v_{\mathrm{fs}}^{2} /\left(\gamma_{\mathrm{snr}}+1\right)$ is the pressure in the SNR forward shock, where $v_{\mathrm{fs}}$ is the speed of the forward shock, and $\rho_{\text {ism }}$ is the number density of the interstellar medium (ISM). Finally, the dynamical evolution radius of the termination shock is estimated by $r_{0}(t)=\sqrt{L(t) / 4 \pi c P_{\mathrm{ts}}(t)}$, where $P_{\mathrm{ts}}(t)$ is the pressure of the gas at the shock (Bucciantini et al. 2004), and the evolution of SNR are calculated with the semi-analytic model provided by Truelove \& McKee (1999).

It should be noted that the SNR photon fields may prevent high energy $\gamma$-rays from leaving the PWN via $\gamma+\gamma \rightarrow e^{+}+$ $e^{-}$(e.g. Murase et al. 2015). In this paper, the $\gamma$-ray attenuation must also be taken into account and the photon fields of SNR are assumed to be a thermal spectrum for simplicity. According to Murase et al. (2015), the optical depth to pair production is approximated as

$\tau_{\gamma \gamma-\mathrm{sn}}=\frac{3}{16} \sigma_{\mathrm{T}} n_{\gamma}^{\mathrm{sn}} R_{\mathrm{ej}}(t) \mathcal{G}(y)$,

where $R_{\mathrm{ej}}(t)$ is the radius of the SNR ejecta, and $n_{\gamma}^{\mathrm{sn}}=$ $2 \zeta(3)\left(k_{\mathrm{B}} T_{\mathrm{sn}}\right)^{3} /\left(\pi^{2} \hbar^{3} c^{3}\right)$ is the photon density of SNR with a temperature of $T_{\mathrm{sn}}$. Here $k_{\mathrm{B}}$ is the Boltzmann constant and $\sigma_{T}$ represents the Thomson cross section. The function $\mathcal{G}(y)=\mathcal{F}(y) / \zeta(3)$ with $y=m_{\mathrm{e}} c^{2} / x k_{\mathrm{B}} T_{\mathrm{sn}}$, in which $\mathcal{F}(y)$ is given by Dermer et al. (2012) and $\zeta(3)=1.202$ is the Riemann's zeta function. In order to consider the $\gamma$-ray attenuation by SNR photon fields, the photon spectrum is modified by multiplying by a factor of $\mathrm{e}^{-\tau_{\gamma \gamma-\mathrm{sn}}}$.

\subsection{Results}

Vela $\mathrm{X}$ is a dynamical system that shows a complex morphology at different observation bands (e.g. Aschenbach et al. 1995; Helfand et al. 2001; Aharonian et al. 2006; Grondin et al. 2013). It is generally believed that the PWN flow has been crushed by the SNR reverse shock, which leads to Vela PWN having a very complex observation morphology (e.g. Abdo et al. 2010; Hinton et al. 2011). With the parameters listed in Table 1, we calculated the dynamical radii of the Vela SNR system, including the radius of the $\mathrm{SNR}\left(R_{\mathrm{SNR}}\right)$, reverse shock $\left(R_{\mathrm{rs}}\right), \mathrm{PWN}\left(R_{\mathrm{pwn}}\right)$, and termination shock $\left(R_{\mathrm{ts}}\right)$ in the framework of our model. The results are shown in Fig. 1, where the stars represent the current radius and termination shock radius of Vela PWN and imply that the nebula is in the phase of reverberation. From the dynamical modelling results, we obtain that the radius of Vela X has a value of $R_{\text {pwn }}\left(T_{\text {age }}\right)=1.25^{\circ}$ and the termination shock radius is $R_{\mathrm{ts}}\left(T_{\text {age }}\right)=37^{\prime \prime}$ at the age of $T_{\text {age }}=11.3 \mathrm{kyr}$, which is consistent with the values $R_{\text {pwn }}\left(T_{\text {age }}\right)=1.2^{\circ}$ shown in Abramowski et al. (2012) and $R_{\mathrm{ts}}\left(T_{\mathrm{age}}\right)=25^{\prime \prime}$ derived by Ng \& Romani (2004).

Since the photon SEDs of Vela $X$ at different energy bands of different emission regions and the radial variations of photon 
Table 1. Values of parameters for Vela X.

\begin{tabular}{lcc}
\hline \hline Pulsar and ejecta parameters & Symbol & Value \\
\hline SN explosion energy $\left(10^{51} \mathrm{erg}\right)$ & $E_{\mathrm{SN}}$ & 1.0 \\
Ejected mass $\left(M_{\odot}\right)$ & $M_{\mathrm{ej}}$ & 8.05 \\
ISM density $\left(\mathrm{cm}^{-3}\right)$ & $\rho_{\mathrm{ism}}$ & 0.1 \\
Period $(\mathrm{ms})$ & $P$ & 89.3 \\
Period derivative $\left(\mathrm{s} \mathrm{s}^{-1}\right)$ & $\dot{P}$ & $1.25 \times 10^{-13}$ \\
Initial spin-down power $\left(\mathrm{erg} \mathrm{s}^{-1}\right)$ & $L_{0}$ & $3.64 \times 10^{37}$ \\
Initial spin-down timescale $(\mathrm{kyr})$ & $\tau_{0}$ & 21.0 \\
Braking index & $n$ & 1.7 \\
Age $(\mathrm{kyr})$ & $T_{\mathrm{age}}$ & 11.3 \\
Distance (pc) & $d$ & 290 \\
\hline Fitted parameters & $\eta_{\mathrm{B}}$ & 0.0087 \\
\hline Magnetic fraction & $\eta_{\mathrm{R}}$ & 0.97 \\
Radio electron fraction & $\eta_{\mathrm{X}}$ & 0.018 \\
X-ray electron fraction & $\alpha_{1}$ & 1.91 \\
Spectral index of radio electrons & $\gamma_{\mathrm{max}, \mathrm{R}}$ & $1.1 \times 10^{6}$ \\
Maximum energy of radio electrons & $2.0 \times 10^{9}$ \\
Maximum energy of X-ray electrons & $\gamma_{\mathrm{max}, \mathrm{X}}$ & $2.0 \times 10^{18}$ \\
Initial diffusion coefficient $\left(\mathrm{cm}{ }^{2} \mathrm{~s}^{-1}\right)$ & $\kappa_{0}$ & $9.0 \times 12$ \\
SNR temperature (K) & $T_{\mathrm{sn}}$ & 42 \\
\hline
\end{tabular}

index in the X-ray band as well as the surface brightness in the $\mathrm{TeV} \gamma$-rays have been observed, all of the observations should be reproduced by our model to obtain the best-fit parameters. In the calculation, we first reproduced the photon SEDs of different emission regions roughly, and then adjusted the parameters of the initial diffusion coefficient and magnetic fraction $\eta_{\mathrm{B}}$ to reproduce the radial variations of the photon index and flux in the X-ray band as well as surface brightness in the TeV $\gamma$-rays, which due to the radial profiles are mainly determined by the competition of synchrotron radiation loss and particle diffusion (e.g. Vorster \& Moraal 2013), and then adjusted the fitted parameters again to reproduce the photon SEDs. We repeated the above steps and we obtained the parameters of Vela X, which are listed in Table 1.

The photon SEDs of Vela X calculated with our model are shown in Fig. 2, where the left panel is the comparison of our model results of the PWN with the observed data from the radio to $\gamma$-ray bands, and the right panel is the comparison of model results with the data observed in the $\gamma$-ray band. The soft photon fields, which include three components in the calculation, are as follows: the cosmic microwave background $\left(\mathrm{CMB}\right.$, temperature $2.73 \mathrm{~K}$, photon density $0.26 \mathrm{eV} \mathrm{cm}^{-3}$ ), the galactic far-infrared background (FIR, temperature $25 \mathrm{~K}$, photon density $12.0 \mathrm{eV} \mathrm{cm}^{-3}$ ), and the near-infrared and optical photon field (NIR) due to the stars (temperature $6500 \mathrm{~K}$, photon density $\left.0.4 \mathrm{eV} \mathrm{cm}^{-3}\right)$. The $\chi^{2}$ value of the model results with the observed data in the $\mathrm{GeV}$ band is 2.2 and in the $\mathrm{TeV}$ for the inner emission region of $r=48$ arcmin we obtain 2.5. The luminosity of Vela $\mathrm{X}$ at different bands of the different emission regions respectively derived from the model and the observations are shown in Table 2, revealing that the luminosities at different bands obtained by our model are consistent with the results of observations with the exception of the $\mathrm{TeV} \gamma$-ray luminosity. The TeV $\gamma$-ray luminosity obtained by our model is less than the result estimated by Aharonian et al. (2006), but the integral flux above $1 \mathrm{TeV}$ derived from our model of $1.9 \times 10^{-11} \mathrm{~cm}^{-2} \mathrm{~s}^{-1}$ is consistent with the value $2.1 \times 10^{-11} \mathrm{~cm}^{-2} \mathrm{~s}^{-1}$ obtained by Abramowski et al. (2012) according to the deeper observation of H.E.S.S.

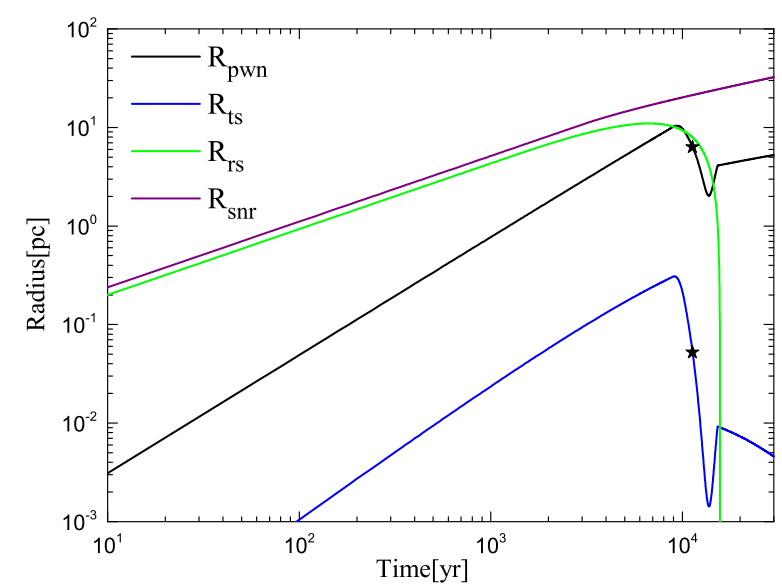

Fig. 1. Dynamical evolution radius of SNR $\left(R_{\mathrm{snr}}\right.$, purple line), reverse shock $\left(R_{\mathrm{rs}}\right.$, green line), PWN ( $R_{\mathrm{pwn}}$, black line) and the termination shock $\left(R_{\mathrm{ts}}\right.$, blue line). The stars represent the current radius and terminate shock radius of Vela PWN at $T_{\text {age }}=11.3 \mathrm{kyr}$.

As seen in Fig. 2, the observed SEDs of Vela X, including $\mathrm{X}$-ray photon spectra of the inner emission regions and $\gamma$-ray spectra in the $\mathrm{GeV}$ and $\mathrm{TeV}$ bands, can be well reproduced in the framework of our model with the parameters shown in Table 1, while the radio photon spectrum cannot. It should be pointed out that we did not find any set of appropriate parameters to reproduce the SED of Vela X in the radio band based on the spherically symmetric spatially dependent model. From the modelling results, we can also see that the process of $\gamma$-ray attenuation by the SNR photon field is important for modifying the VHE $\gamma$-ray spectrm of Vela X (see the right panel of Fig. 2), and a temperature of $T_{\mathrm{sn}}=42 \mathrm{~K}$ of Vela SNR photon field is obtained. We note that such a photon field around the nebula has not been detected by the current observations of Vela $\mathrm{X}$, but this value is interestingly consistent with the supernova $(\mathrm{SN})$ formed dust temperature for the young PWNe of 50-60 K estimated by Temim et al. (2012).

The photon field produced by synchrotron emission of the PWN also may prevent high energy photons from leaving the nebula. In order to study the effect of photon-photon annihilation of the synchrotron photon inside the PWN on the photon SEDs, we calculated the optical depths to the pair production with the parameters listed in Table 1 and the results are given in Fig. 3, where the optical depth to the SNR photon field is calculated with Eq. (12) and the optical depth to the synchrotron photon field of the PWN are determined by (e.g. Aharonian et al. 2008)

$\tau_{\gamma \gamma-\mathrm{pwn}}=\int_{r_{0}}^{R_{\mathrm{pwn}}} \int_{0}^{\infty} \sigma_{\gamma \gamma} n_{\gamma}(r, x, t) \mathrm{d} x \mathrm{~d} r$

where $n_{\gamma}(r, x, t)$ is the number density of non-thermal photons and $\sigma_{\gamma \gamma}$ represents the photon-photon annihilation cross section. It can be seen from Fig. 3 that the process of photon-photon annihilation of synchrotron photons inside the PWN can be neglected due to the optical depth $\tau_{\gamma \gamma-\text { pwn }} \ll \tau_{\gamma \gamma-\text { sn }}$, and that the VHE $\gamma$-ray spectrum of Vela $\mathrm{X}$ is mainly modified by the photon-photon annihilation process of the SNR photon field.

To analyse the effect of the processes of synchrotron selfabsorption and pair production on the photon spectrum, we also calculated the timescales of photon escape, synchrotron selfabsorption, and pair production inside the PWN. The results are shown in Fig. 4. In the calculation, the photon escape timescale is calculated with $t_{\mathrm{esc}}=R_{\mathrm{pwn}}(t) / c$, the timescale of photon-photon 

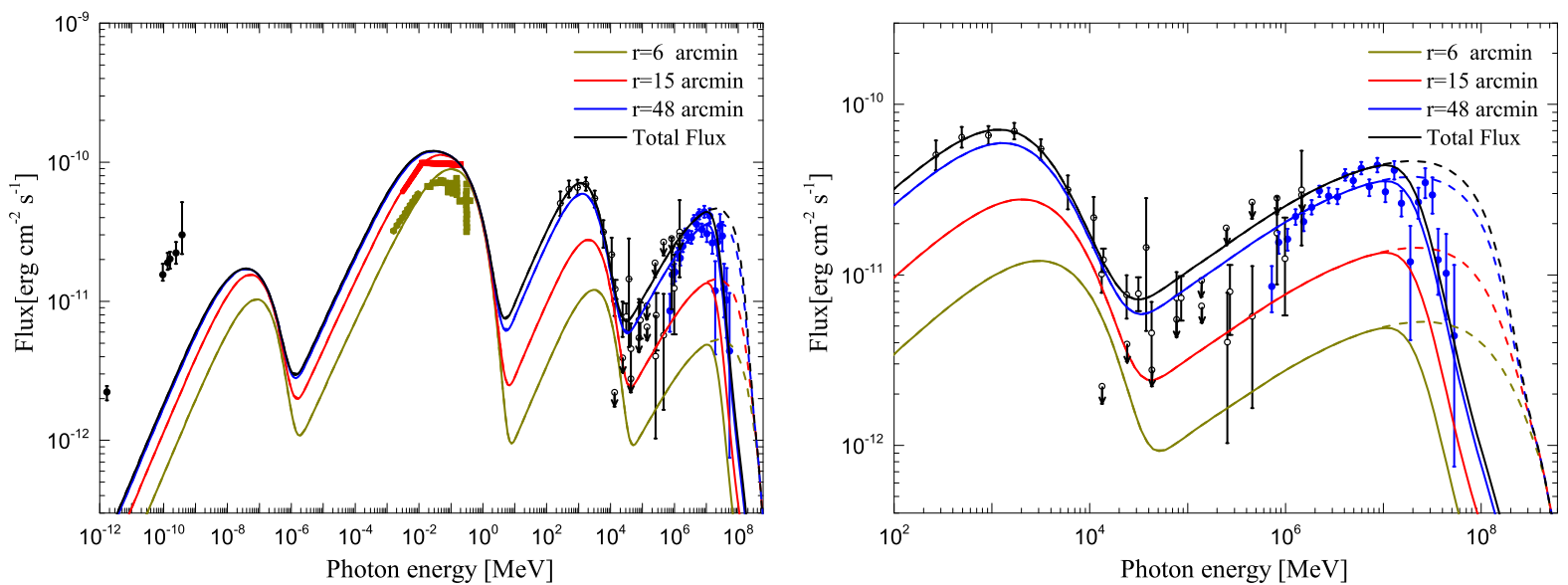

Fig. 2. Comparison of modelling results of Vela $\mathrm{X}$ with the observed data at different emission regions of $r=6 \operatorname{arcmin}$ (dark-yellow line), $r=15 \mathrm{arcmin}$ (red line), and $r=48$ arcmin (blue line), as well as the total flux (black line). Left panel: modelling SEDs from radio to $\gamma$-ray bands. Right panel: modelling SEDs in the $\gamma$-ray band. The dash-dotted lines are the unabsorbed spectra, and the solid lines consider $\gamma$-ray attenuation by the SNR photon fields. The observed data are taken from Abdo et al. (2010) for radio band (black, full circles), Mattana et al. (2011) and Mangano et al. (2005) for X-rays of the inner regions of $r=6$ arcmin (dark-yellow, solid thick line) and $r=15$ arcmin (red, solid thick line), Grondin et al. (2013), Ajello et al. (2017), and Tibaldo et al. (2018) for GeV $\gamma$-rays (black, open circles), and Abramowski et al. (2012) for TeV $\gamma$-rays emission within $r=48$ arcmin (blue, full circles), respectively.

Table 2. Values derived for the luminosity of Vela X.

\begin{tabular}{ccccl}
\hline \hline Radius of emission region & Energy band & $L_{\text {model }}\left(10^{33} \mathrm{erg} \mathrm{s}^{-1}\right)$ & $L_{\mathrm{obs}}\left(10^{33} \mathrm{erg} \mathrm{s}^{-1}\right)$ & Origin \\
\hline $6^{\prime}$ & $20-200 \mathrm{keV}$ & 1.2 & 1.1 & Mattana et al. (2011) \\
$15^{\prime}$ & $0.1-200 \mathrm{keV}$ & 5.3 & 5.5 & Mangano et al. (2005) \\
$75^{\prime}$ & $0.2-100 \mathrm{GeV}$ & 2.3 & 2.4 & Grondin et al. (2013) \\
$75^{\prime}$ & $0.55-65 \mathrm{TeV}$ & 0.87 & 0.99 & Aharonian et al. (2006) \\
\hline
\end{tabular}

Notes. The symbols $L_{\mathrm{obs}}$ and $L_{\text {model }}$ represent the luminosity at different bands derived from the observations and the model, respectively.

pair production $t_{\gamma \gamma}$ inside the PWN is calculated by Eq. (28) of Lu et al. (2017a), and the synchrotron self-absorption timescale is given by (e.g. Mastichiadis \& Kirk 1995)

$t_{\mathrm{ssa}}=\left[-\sigma_{\mathrm{T}} c \frac{\pi}{6 \alpha_{f}} x^{-1 / 2} b^{-1 / 2}\left[\frac{\partial}{\partial \gamma}\left(\frac{n_{\mathrm{e}}}{\gamma^{2}}\right)\right]_{\gamma=(x / b)^{1 / 2}}\right]^{-1}$,

where $\alpha_{\mathrm{f}}$ is the fine-structure constant and $b=B(r, t) / B_{\mathrm{cr}}$ with the critical magnetic field $B_{\mathrm{cr}}$. The results reveal that although the processes of synchrotron self-absorption and pair production inside the PWN are considered in the model, they can be neglected because of the timescales of $t_{\gamma \gamma} \ll t_{\mathrm{esc}}$ and $t_{\mathrm{ssa}} \ll t_{\mathrm{esc}}$ in all of the emission regions. Therefore, the processes of synchrotron self-absorption and pair production inside the PWN only have little effect on the photon spectrum of Vela X.

With the parameters listed in Table 1, the high energy radiative spectra of Vela $\mathrm{X}$ are well reproduced at the age of $11.3 \mathrm{kyr}$. At this age, the present-day spin-down luminosity of the Vela pulsar is estimated to be $6.9 \times 10^{36} \mathrm{erg} \mathrm{s}^{-1}$, which is consistent with the value given by Taylor et al. (1993). According to the model results, a fraction of pulsar spin-down power converted to magnetic energy is derived to be $\eta_{\mathrm{B}}=0.0087$, which implies that the current magnetic field of Vela $X$ at the termination shock has a value of $B_{0}\left(T_{\text {age }}\right)=11.8 \mu \mathrm{G}$ and a spatial average of $\bar{B}\left(T_{\text {age }}\right)=8.7 \mu \mathrm{G}$. The averaged magnetic field strength in our model is larger than the values of $5 \mu \mathrm{G}$ obtained by de Jager et al. (2008) with a spatially independent model and $5.8 \mu \mathrm{G}$ given by Porth et al. (2016) with a steadystate particle transport model. Moreover, with an initial dif-

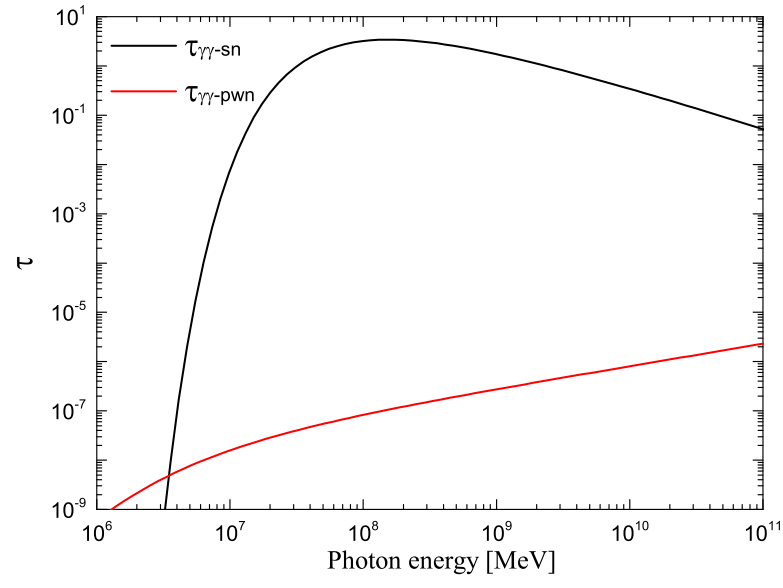

Fig. 3. Present-day optical depths to pair production of synchrotron photon field of the PWN (red line) and SNR photon field (black line), with the parameters listed in Table 1.

fusion coefficient of $9.0 \times 10^{18} \mathrm{~cm}^{2} \mathrm{~s}^{-1}$, the current diffusion coefficient of Vela $X$ at the termination shock is estimated to be $\kappa_{0}\left(T_{\text {age }}\right)=6.13 \times 10^{23} \mathrm{~cm}^{2} \mathrm{~s}^{-1}$ and the spatial averaged diffusion coefficient has a value of $\bar{\kappa}\left(T_{\mathrm{age}}\right)=1.18 \times 10^{24} \mathrm{~cm}^{2} \mathrm{~s}^{-1}$ with an electron energy of $1 \mathrm{TeV}$. These results are smaller than the values $\kappa_{0}\left(T_{\text {age }}\right)=\bar{\kappa}\left(T_{\text {age }}\right)=1.4 \times 10^{26} \mathrm{~cm}^{2} \mathrm{~s}^{-1}$ obtained by Porth et al. (2016), because the particle diffusion is energy independent in their model while it is energy dependent in our model. 

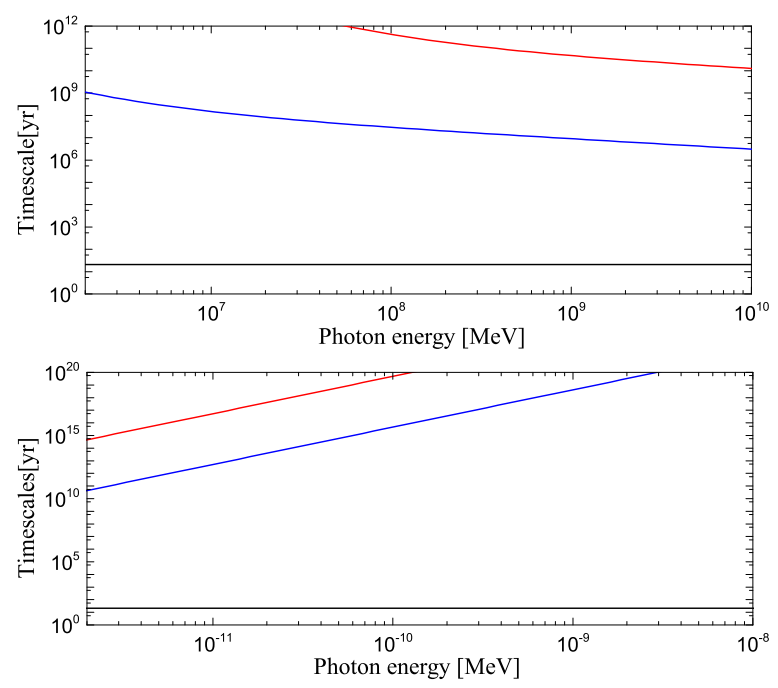

Fig. 4. Current timescales of photon escape (black lines), pair production (upper panel), and synchrotron self-absorption (bottom panel) inside the PWN at different radial distances of $r=3.9$ arcmin (blue lines) and $r=66.8$ arcmin (red lines).

The X-ray observations revealed that the photon index and flux density of Vela $\mathrm{X}$ in the $3-10 \mathrm{keV}$ energy range increase with the increase of radial distance (Mangano et al. 2005). Thus, we calculated the variations of photon index and flux density with radial distance for the nebula according to the parameters listed in Table 1 and the results are shown in Fig. 5, where the fit residual is calculated with residual=(observation-model $) /$ model. In the calculation, the observed data in the energy range of $3-10 \mathrm{keV}$ of the emission region within $r=15 \mathrm{arcmin}$ of the PWN are obtained from Mangano et al. (2005). It can be seen from Fig. 5 that the radial variations of photon index and flux density in this energy range can be well reproduced in the framework of our model when the emission regions are farther away from the central pulsar. While in the vicinity of the pulsar, the observed data cannot be reproduced well with our model, especially for the radial variations of the flux density, which have significant deviations between the data and the model. From the right panel of Fig. 5, it is clear that the flux density calculated with our model is less than the observed flux density of the inner emission regions. On the one hand, the particle distribution in the nebula is assumed to be isotropic and a toroidal magnetic field is adopted in our model, while the particle and magnetic field in the PWN may have more complicated distributions (e.g. Del Zanna et al. 2004; Porth et al. 2014). On the other hand, it could be that the observed data of the emission regions in the vicinity of the pulsar include photons that emit from the central pulsar, which leads to higher values of the observed flux density. The modelling results also show that the photon index and flux density of Vela X in the X-ray band have more significantly radial variations in the vicinity of the Vela pulsar, because the radial variations of magnetic field and diffusion in the inner regions are more obvious (Kennel \& Coroniti 1984a; Lu et al. 2017b), and the SED shape in the X-ray band is determined by the competition between the radiation losses and particle diffusion (Vorster \& Moraal 2013).

Based on deeper observations of the entire Vela X nebula region with H.E.S.S., Abramowski et al. (2012) have shown that the energy averaged surface brightness of the $\gamma$-ray emission of the nebula in the $0.75-70 \mathrm{TeV}$ energy range has obvious changes as the radial distance increases. We calculated the differential $\gamma$-ray spectra of the inner region of $r=48 \mathrm{arcmin}$ and ring extension of $48<r<72$ arcmin of Vela X, as well as the averaged surface brightness in the $0.75-70 \mathrm{TeV}$ energy range with the same model parameters. The results are shown in Fig. 6 and the observed data are taken from Abramowski et al. (2012). From the left panel of Fig. 6, it is clear that the $\gamma$-ray spectra of the inner region and ring extension of Vela $X$ can be well reproduced in our model. The comparison of radial variations of the energy averaged surface brightness of the PWN in the $0.75-70 \mathrm{TeV}$ energy range are shown in the right panel of Fig. 6, indicating that our model can also reproduce the decreased surface brightness. In our model, the nebula is assumed to be a spherical symmetric system and the particle distribution inside the nebula is considered to be isotropic, while the $\mathrm{TeV} \gamma$-ray observations of H.E.S.S. revealed that Vela $\mathrm{X}$ is an asymmetric PWN, which leads the radial variations of surface brightness in the $\mathrm{TeV}$ band to have some deviations between the observations and the model.

The central position of VHE $\gamma$-ray emission of Vela $\mathrm{X}$ is not located at the location of the Vela pulsar because the view fields in the $\gamma$-rays revealed that the significant emissions are mainly centred on the south of the pulsar (Aharonian et al. 2006; Abramowski et al. 2012), which is different from that shown in the X-ray band. The observations have shown that the central position of X-ray emission is located at the location of the central pulsar (Mangano et al. 2005). We also note that the central position of $\mathrm{GeV} \gamma$-ray emission is also not located at the location of the Vela pulsar but located at the south of the pulsar, and that it is not the same as the central position of the $\mathrm{TeV} \gamma$-ray emission because the observed image in the $\mathrm{GeV}$ band is different from that in the TeV band (e.g. Abdo et al. 2010; Tibaldo et al. 2018). In our model, the particles are injected from the central pulsar, that is, the central position of photon emission is located at the location of the central pulsar. Therefore, the $\mathrm{TeV} \gamma$-ray spectra of the inner region and ring extension of Vela $X$, as well as the radial variations of surface brightness in $0.75-70 \mathrm{TeV}$ energy range, can be reproduced by our model, but they are somewhat different from the observations. In order to investigate the spatial radiative properties of Vela $\mathrm{X}$ more accurately at different bands, a non-spherically symmetric spatially dependent model that involves the proper motion of the pulsar should be presented, though it is outside of the scope of our paper.

Abramowski et al. (2012) have pointed out that the energy spectrum of Vela $\mathrm{X}$ in the $0.75-70 \mathrm{TeV}$ energy range can be well described by an exponentially cut-off power-law function, $\mathrm{d} N / \mathrm{d} E \propto(E / 1 \mathrm{TeV})^{-\Gamma} e^{-E / E_{\mathrm{cut}}}$, where $E$ is the energy of photons, $\Gamma$ is the power-law photon index, and $E_{\text {cut }}$ is the cut-off energy. In order to investigate the radial variations of the spectrum in the $\mathrm{TeV}$ band, we also calculated the photon index and cut-off energy of Vela $\mathrm{X}$ in the $0.75-70 \mathrm{TeV}$ energy range with an exponentially cut-off power-law function, and the results are shown in Fig. 7. It can be seen from the left panel of Fig. 7 that the power-law photon index in the $0.75-70 \mathrm{TeV}$ energy range remains almost unchanged as the radial distance increases. The radial variations of the cut-off energy are shown in the right panel of Fig. 7, showing that the cut-off energy decreases with the increase of radial distance in the inner regions, while there are only slight changes in the outer regions. In fact, the shape of the photon spectrum in the $\mathrm{TeV}$ band is determined by the competition between the radiation losses and particle diffusion (Vorster \& Moraal 2013; Lu et al. 2016). In our model, the magnetic field decreases while the diffusion coefficient increases with the increasing time (see Eqs. (6) and (7)). For Vela X with an age of $11.3 \mathrm{kyr}$, the current competition between the radiation 

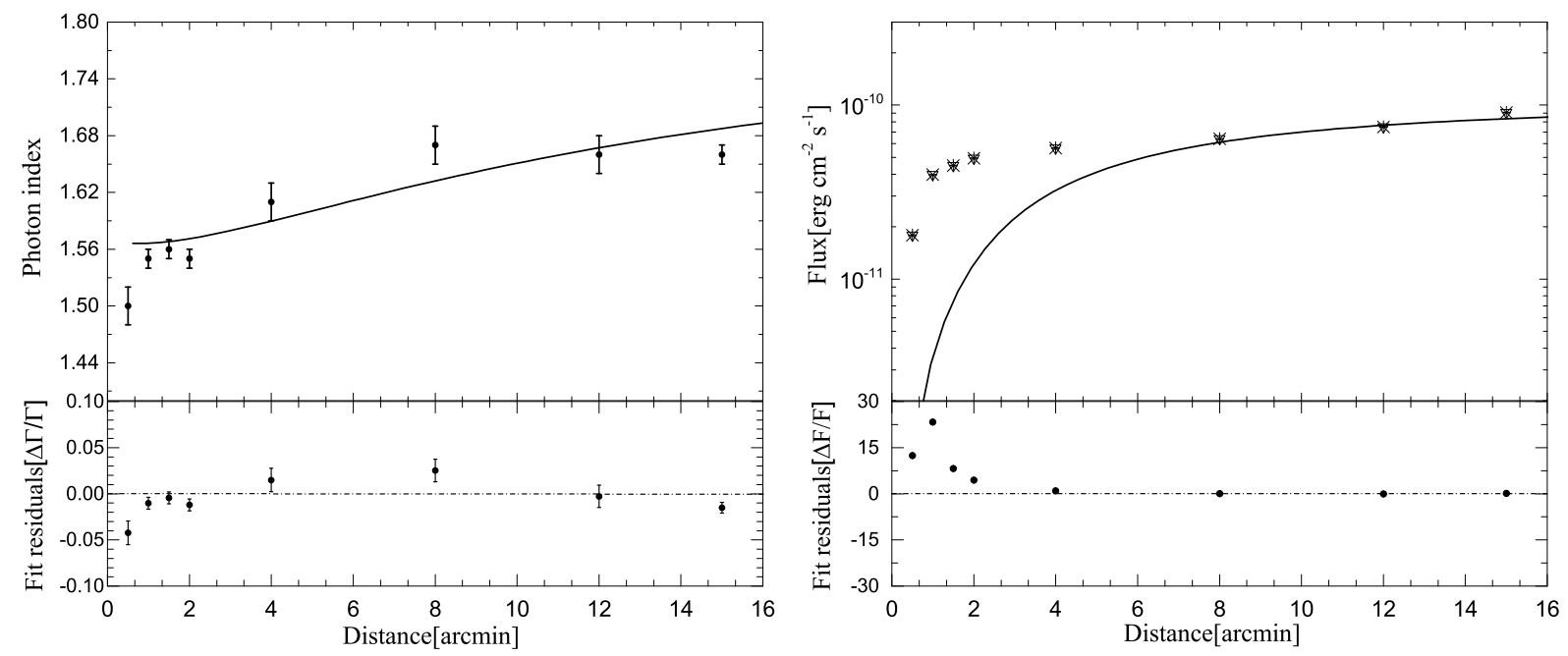

Fig. 5. Comparisons of radial variations of photon index (left panel) and flux density (right panel) in the energy range of 3-10 keV of the Vela X emission within $r=15$ arcmin. The observed data are taken from Mangano et al. (2005).
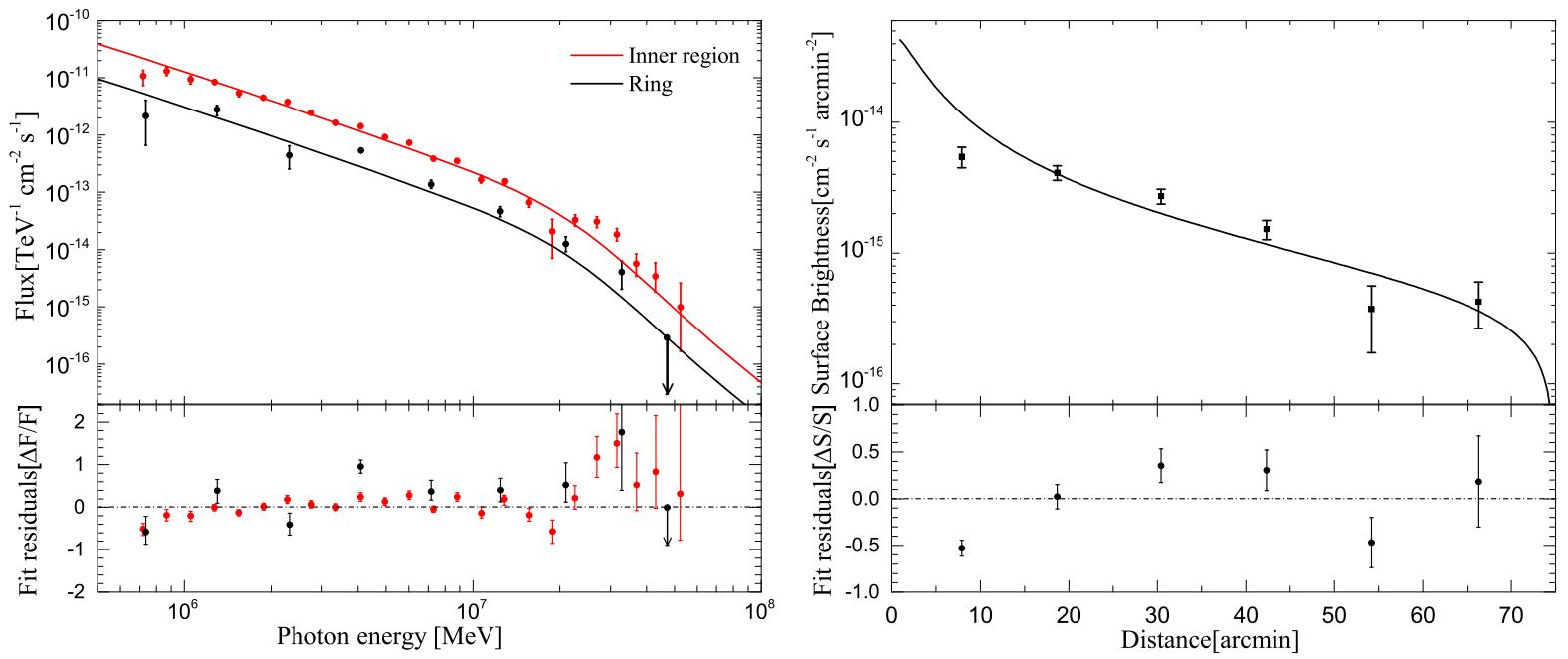

Fig. 6. Left panel: comparisons of differential $\gamma$-ray spectrum of the inner region of $r=48$ arcmin (red line) and ring extension of $48<r<$ 72 arcmin (black line) of Vela $\mathrm{X}$ in the $\mathrm{TeV}$ band. Right panel: radial variations of surface brightness in the $0.75-70 \mathrm{TeV}$ energy range. The observed data are taken from Abramowski et al. (2012).

losses and particle diffusion has transitioned to a diffusiondominated regime. Particle diffusion is important for reducing the amount of radiation losses (Vorster \& Moraal 2013), which leads to the radial variations of photon index and cut-off energy in the TeV energy range as shown in Fig. 7.

Finally, we calculated the radial variations of the power-law photon index and surface brightness of Vela $\mathrm{X}$ in the $0.2-20 \mathrm{GeV}$ energy range, and the results are shown in Fig. 8. Abdo et al. (2010) have pointed out that the total $\mathrm{GeV} \gamma$-ray spectrum in the energy range of $0.2-20 \mathrm{GeV}$ can be well described by a powerlaw function with a photon index of $2.41 \pm 0.09 \pm 0.15$. Thus, the radial photon index of our model in the GeV energy range is also calculated with a power-law function. From the left panel of Fig. 8, it can be seen that the photon index of the inner regions of Vela $\mathrm{X}$ in the $\mathrm{GeV}$ band has a significant increase as the radial distance increases, while there is only a slight changes in the outer regions. In the calculation, the injected particles of Vela X are composed of two distinct electron populations (see Eq. (3)) and $\mathrm{GeV} \gamma$-rays are produced by the lower energy population, indicating that the power-law photon index in the $0.2-20 \mathrm{GeV}$ energy range is also determined by the competition between the radiation losses and particle diffusion. In our model, the particle diffusion is energy dependent and the lower energy particles have a slow diffusion, which give rise to the radial variations of the photon index of Vela $\mathrm{X}$ in the $\mathrm{GeV}$ band being mainly determined by the radiation losses and leading to the variations of photon index in the $0.2-20 \mathrm{GeV}$ energy range as shown in the left panel of Fig. 8. We would like to point out that the radial variations of the photon index of Vela $\mathrm{X}$ in the $\mathrm{GeV}$ band is different from the results of MSH 15-52, G21.5-0.9, and 3C58 obtained by Lu et al. (2017a,b). This is because the injected particles of Vela $\mathrm{X}$ are assumed to be composed of two distinct electron populations, while a broken power-law form is assumed for MSH 15-52, G21.5-0.9, and 3C58 in Lu et al. (2017a,b). The radial variations of surface brightness in the $0.2-20 \mathrm{GeV}$ energy range are given in the right panel of Fig. 8. Similar to the radial variations of energy averaged surface brightness in the $\mathrm{TeV}$ band, the surface brightness of Vela $\mathrm{X}$ in the GeV energy range also decreases with the increase of radial distance. It should be pointed out that although the Fermi-LAT observations have 

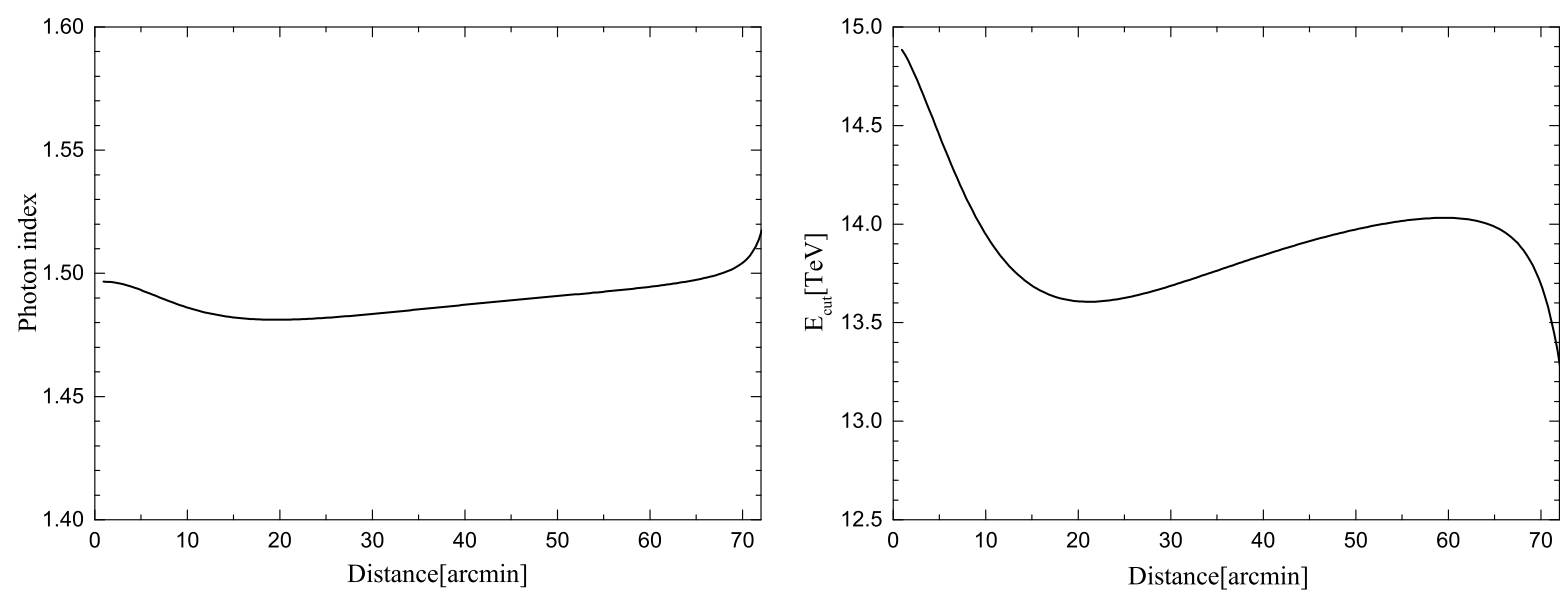

Fig. 7. Radial variations of photon index (left panel) and cut-off energy (right panel) of Vela X in the $0.75-70 \mathrm{TeV}$ energy range.
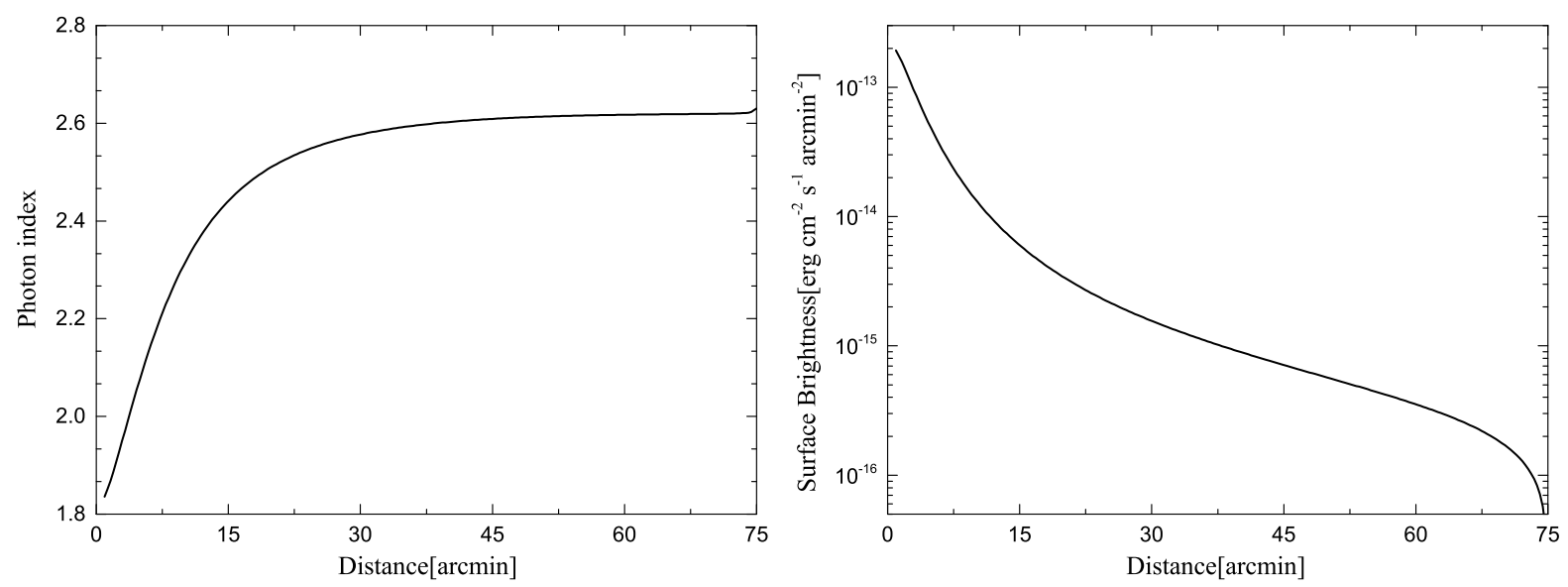

Fig. 8. Radial variations of power-law photon index (left panel) and surface brightness (right panel) of Vela X in the energy range of $0.2-20 \mathrm{GeV}$.

revealed that the photon spectra of Vela $X$ in the southern and northern wings have some differences (Grondin et al. 2013), the spatial variations of photon index and surface brightness at the $\mathrm{GeV}$ band have not been reported in the literature. Therefore, the spatial variations of Vela $\mathrm{X}$ in the $\mathrm{GeV}$ band predicted by our model should be tested in the further observations.

\section{Summary and discussion}

In this paper, non-thermal photon emission and high energy spatially radiative properties of Vela $\mathrm{X}$ have been investigated in the framework of a spatially dependent particle transport model. The main difference between the model presented here and that given by $\mathrm{Lu}$ et al. (2017a) is that the injected particles are assumed to consist of two distinct electron populations and the $\gamma$-ray attenuation by the SNR photon fields is taken into account.

With the parameters listed in Table 1, the photon SEDs of Vela $\mathrm{X}$ observed at X-ray, $\mathrm{GeV}$, and $\mathrm{TeV} \gamma$-ray bands can be reproduced well in this model (see Fig. 2), and the process of $\gamma$-ray attenuation by the SNR photon fields is important for correcting the VHE $\gamma$-ray spectrum of Vela X (see right panel of Fig. 2), and the temperature of Vela SNR photon field is estimated to be $42 \mathrm{~K}$.

The spatial variations of photon SEDs of Vela $\mathrm{X}$ also have been investigated. From our calculations, on the one hand, the radial variations of photon index and flux density of the emission region within $r=15 \operatorname{arcmin}$ of Vela $\mathrm{X}$ in the $3-10 \mathrm{keV}$ energy range are well reproduced in the model, with the exception of the data in the regions of the vicinity of the central pulsar (see Fig. 5). The difference between model results and the observed data may be that the observed data of the vicinity of the pulsar include the photons whose emission from the central pulsar and the particle distribution in the nebula is assumed to be isotropic and a toroidal magnetic filed is adopted in our model, while the particle and magnetic field in the PWN probably have more complicated distributions. On the other hand, the differential $\gamma$-ray spectra of the inner region of $r=48^{\prime}$ and ring extension of $48^{\prime}<r<72^{\prime}$ as well as the radial variations of averaged surface brightness of Vela $\mathrm{X}$ in the $0.75-70 \mathrm{TeV}$ energy range can be reproduced well in the model (see Fig. 6).

The radial variations of the $\gamma$-ray spectrum in the 0.75-70 TeV energy range have been studied with an exponentially cut-off power-law function, indicating that the photon index of Vela $\mathrm{X}$ in the TeV band only has a slight change as the radial distance increases, and the cut-off energy decreases with the increase of radial distance in the inner regions but only has a slight change in the outer regions (see Fig. 7). Furthermore, the radial variations of the power-law photon index and surface brightness of Vela $\mathrm{X}$ in the $0.2-20 \mathrm{GeV}$ energy range are also predicted, and the results show that the power-law photon index increases while the surface brightness decreases with the increase of radial distance (see Fig. 8). We note that the increased photon index of Vela $\mathrm{X}$ in the $\mathrm{GeV}$ band is different from the results of MSH 15-52, G21.5-0.9, and 3C58 obtained by 
Lu et al. (2017a,b), because the injected particles of Vela $\mathrm{X}$ are assumed to be composed of two distinct electron populations and $\mathrm{GeV} \gamma$-rays are produced by the lower energy population.

It should be pointed out that the particles in the model are injected from the central pulsar, which leads to the central position of photon emission of our model being located at the location of the pulsar. The observations showed that the central positions of $\mathrm{GeV}$ and $\mathrm{TeV} \gamma$-ray emissions of Vela $\mathrm{X}$ are located at the south of the Vela pulsar (Aharonian et al. 2006; Abdo et al. 2010; Abramowski et al. 2012; Grondin et al. 2013; Tibaldo et al. 2018), and the central positions shown in the GeV and $\mathrm{TeV}$ bands are different. Therefore, although our model can roughly account for the high energy spatially radiative properties of Vela X, a non-spherically symmetric spatially dependent model, which includes the proper motion of the pulsar, should be presented to investigate the radiative properties of the PWN more precisely.

Acknowledgements. We are very grateful to the anonymous referee for his or her very constructive comments. This work is partially supported by the National Natural Science Foundation of China (NSFC 11433004, U1738211, 11173020, 11803027), Top Talents Program of Yunnan Province, and Yunnan local colleges applied basic research projects (2017FH001-17).

\section{References}

Abdo, A. A., Ackermann, M., Ajello, M., et al. 2010, ApJ, 713, 146 Abramowski, A., Acero, F., Aharonian, F., et al. 2012, A\&A, 548, A38

Aharonian, F., Akhperjanian, A. G., Bazer-Bachi, A. R., et al. 2006, A\&A, 448, L43

Aharonian, F. A., Khangulyan, D., \& Costamante, L. 2008, MNRAS, 387, 1206

Ajello, M., Atwood, W. B., Baldini, L., et al. 2017, ApJS, 232, 18

Alvarez, H., Aparici, J., May, J., \& Reich, P. 2001, A\&A, 372, 636

Aschenbach, B., Egger, R., \& Trümper, J. 1995, Nature, 373, 587

Bucciantini, N., Bandiera, R., Blondin, J. M., Amato, E., \& Del Zanna, L. 2004 A\&A, 422, 609

Bucciantini, N., Arons, J., \& Amato, E. 2011, MNRAS, 410, 381

Caraveo, P. A., De Luca, A., Mignani, R. P., \& Bignami, G. F. 2001, ApJ, 561, 930

de Jager, O. C., Slane, P. O., \& LaMassa, S. 2008, ApJ, 689, L125
Del Zanna, L., Amato, E., \& Bucciantini, N. 2004, A\&A, 421, 1063

Dermer, C. D., Murase, K., \& Takami, H. 2012, ApJ, 755, 147

Dodson, R., Legge, D., Reynolds, J. E., \& McCulloch, P. M. 2003, ApJ, 596, 1137

Espinoza, C. M., Lyne, A. G., \& Stappers, B. W. 2017, MNRAS, 466, 147

Grondin, M.-H., Romani, R. W., Lemoine-Goumard, M., et al. 2013, ApJ, 774, 110

Helfand, D. J., Gotthelf, E. V., \& Halpern, J. P. 2001, ApJ, 556, 380

Hinton, J. A., Funk, S., Parsons, R. D., \& Ohm, S. 2011, ApJ, 743, L7

Holler, M., Schöck, F. M., Eger, P., et al. 2012, A\&A, 539, A24

Horns, D., Aharonian, F., Santangelo, A., Hoffmann, A. I. D., \& Masterson, C. 2006, A\&A, 451, L51

Ishizaki, W., Tanaka, S. J., Asano, K., \& Terasawa, T. 2017, ApJ, 838, 142

Kennel, C. F., \& Coroniti, F. V. 1984a, ApJ, 283, 694

Kennel, C. F., \& Coroniti, F. V. 1984b, ApJ, 283, 710

LaMassa, S. M., Slane, P. O., \& de Jager, O. C. 2008, ApJ, 689, L121

Large, M. I., Vaughan, A. E., \& Mills, B. Y. 1968, Nature, 220, 340

Lerche, I., \& Schlickeiser, R. 1981, ApJS, 47, 33

Lu, F.-W., Gao, Q.-G., \& Zhang, L. 2016, Res. Astron. Astrophys., 16, 90

Lu, F.-W., Gao, Q.-G., \& Zhang, L. 2017a, ApJ, 834, 43

Lu, F.-W., Gao, Q.-G., Zhu, B.-T., \& Zhang, L. 2017b, MNRAS, 472, 2926

Lyne, A. G., Pritchard, R. S., Graham-Smith, F., \& Camilo, F. 1996, Nature, 381, 497

Mangano, V., Massaro, E., Bocchino, F., Mineo, T., \& Cusumano, G. 2005, A\&A, 436, 917

Manzali, A., De Luca, A., \& Caraveo, P. A. 2007, ApJ, 669, 570

Markwardt, C. B., \& Ögelman, H. 1995, Nature, 375, 40

Markwardt, C. B., \& Ögelman, H. B. 1997, ApJ, 480, L13

Mastichiadis, A., \& Kirk, J. G. 1995, A\&A, 295, 613

Mattana, F., Götz, D., Terrier, R., et al. 2011, ApJ, 743, L18

Murase, K., Kashiyama, K., Kiuchi, K., \& Bartos, I. 2015, ApJ, 805, 82

Ng, C. K., \& Gleeson, L. J. 1975, Sol. Phys., 43, 475

Ng, C.-Y., \& Romani, R. W. 2004, ApJ, 601, 479

Pacini, F., \& Salvati, M. 1973, ApJ, 186, 249

Porth, O., Komissarov, S. S., \& Keppens, R. 2014, MNRAS, 438, 278

Porth, O., Vorster, M. J., Lyutikov, M., \& Engelbrecht, N. E. 2016, MNRAS, 460, 4135

Reichley, P.E., Downs, G.S., \& Morris, G.A. 1970, ApJ, 159,

Taylor, J. H., Manchester, R. N., \& Lyne, A. G. 1993, ApJS, 88, 529

Temim, T., Slane, P., Arendt, R. G., \& Dwek, E. 2012, ApJ, 745, 46

Tibaldo, L., Zanin, R., Faggioli, G., et al. 2018, A\&A, 617, A78

Truelove, J. K., \& McKee, C. F. 1999, ApJS, 120, 299

van Rensburg, C., Krüger, P. P., \& Venter, C. 2018, MNRAS, 477, 3853

Vorster, M. J., \& Moraal, H. 2013, ApJ, 765, 30

Weiler, K. W., \& Panagia, N. 1980, A\&A, 90, 269

Zhang, L., \& Yang, X. C. 2009, ApJ, 699, L153 